\title{
A remark on the distribution of products of independent normal random variables
}

\author{
Jerzy Szczepański* \\ a Jagiellonian Universtity, Faculty of Mathematics and Computer Science, ul. S. Łojasiewicza 6, 30-348 Kraków, Poland
}

\section{Article history}

Received: 6 January 2021 Received in revised form: 8 March 2021

Accepted: 9 March 2021

Available: 10 March 2021

\begin{abstract}
We present a proof of the explicit formula of the probability density function of the product of normally distributed independent random variables using the multiplicative convolution formula for Meijer G functions.
\end{abstract}

Keywords: normal distribution, Meijer G-functions

\section{Introduction}

Basic statistic tests are constructed with the aim of sums $\xi_{1}+\xi_{2}+\cdots+\xi_{n}$, squares $\xi_{1}^{2}, \xi_{2}^{2}, \ldots, \xi_{n}^{2}$, sum of squares $\xi_{1}^{2}+\xi_{2}^{2}+\cdots+\xi_{n}^{2}$, quotients $\frac{\xi_{1}}{\xi_{2}}$ or other algebraic operations on sequences of independent random variables $\xi_{1}, \xi_{2}, \ldots, \xi_{n}$ (see eg. [6]). While the sum $\xi_{1}+\xi_{2}+\cdots+\xi_{n}$ has a density that is commonly easy to determine other algebraic operations lead to densities that are more and more complicated and demand special functions in analysis. For example the sum of the independent identically distributed (abbreviated as i.i.d.) random variables $\xi_{i}, i=1,2, \ldots, n$ with the normal $N\left(m_{i}, \sigma_{i}\right)$ probability density function (p.d.f. for abbreviation)

$$
f_{i}(t):=\frac{1}{\sigma_{i} \sqrt{2 \pi}} \exp \left(-\frac{1}{2}\left(\frac{t-m_{i}}{\sigma_{i}}\right)^{2}\right)
$$

has p.d.f. of the same family of normal distributions $N(m, \sigma)$

$$
f(t):=\frac{1}{\sigma \sqrt{2 \pi}} \exp \left(-\frac{1}{2}\left(\frac{t-m}{\sigma}\right)^{2}\right)
$$

*Corresponding author: Jerzy.Szczepanski@uj.edu.pl 
with $m:=m_{1}+m_{2}+\cdots+m_{n}$ and $\sigma^{2}=\sigma_{1}^{2}+\sigma_{2}^{2}+\cdots+\sigma_{n}^{2}$. The sum

$$
\xi_{1}^{2}+\xi_{2}^{2}+\cdots+\xi_{n}^{2}
$$

of i.i.d. random variables with standard normal p.d.f. (i.e. $N(m, \sigma)$ with $m=0$ and $\sigma=1)$ is the $\chi_{n}^{2}$ distribution with $n$ degrees of freedom, i.e. has p.d.f. defined by

$$
f(t):= \begin{cases}\frac{1}{2^{\frac{n}{2}} \Gamma\left(\frac{n}{2}\right)} t^{\frac{n}{2}-1} e^{-\frac{t}{2}}, & t \geq 0 \\ 0, & t<0\end{cases}
$$

which requires the use of the Euler gamma function $\Gamma$. It is known (see [9], [10]) that the answer to a very simple question about the distribution of the product of normally distributed random variables requires the use of the Meijer $G$-functions $G_{0, m}^{m, 0}$, see (2). We present a proof of the explicit formula of the probability density function of the product of normally distributed independent random variables in terms of Meijer $G$ functions using the multiplicative convolution formula for Meijer $G$-functions (8). In some special cases the function $G_{p, q}^{m, n}$ can be reduced to a simpler form (see [1]), for example standard normal distribution $N(0,1)$ has the p.d.f. given by

$$
f(t)=\frac{1}{\sqrt{2 \pi}} \exp \left(-\frac{t^{2}}{2}\right)=\frac{1}{\sqrt{2 \pi}} G_{0,1}^{1,0}\left(\frac{t^{2}}{2} \mid \begin{array}{ll}
- & - \\
0 & -
\end{array}\right)
$$

The function $G_{0,2}^{2,0}(x)$ can be expressed with the modified Bessel function of the second kind $K_{\nu}$, see (11).

\section{Meijer G-functions and their basic properties}

The $G$ - function was introduced by C.S. Meijer in 1936 and is defined in terms of Mellin-Barnes type integrals (see [1], [2] and references given there)

$$
G_{p, q}^{m, n}\left(z \mid \begin{array}{l}
a \\
b
\end{array}\right)=\frac{1}{2 \pi i} \int_{L} \frac{\prod_{k=1}^{m} \Gamma\left(b_{k}-\zeta\right)}{\prod_{k=m+1}^{q} \Gamma\left(1-b_{k}+\zeta\right)} \frac{\prod_{k=1}^{n} \Gamma\left(1-a_{k}+\zeta\right)}{\prod_{k=n+1}^{p} \Gamma\left(a_{k}-\zeta\right)} z^{\zeta} d \zeta
$$

where $a=\left(a_{1}, \ldots, a_{n}, a_{n+1}, \ldots, a_{p}\right)$ and $b=\left(b_{1}, \ldots, b_{m}, b_{m+1}, \ldots, b_{q}\right)$ are sequences of real or complex parameters, with the contour of integration $L$ suitably chosen on the complex plane $\mathbb{C}$. If $m=0, n=0, p=0$ or $q=0$ we put 1 in place of the product over an empty set of indices and we put a sign of omission ' - ' in 
an appropriate place in the symbol of the $G$-function or omitting the sign '-' if this does not lead to confusion. For example

$$
\begin{aligned}
G_{0, m}^{m, 0}\left(z \mid b_{1}, b_{2}, \ldots, b_{m}\right) & =G_{0, m}^{m, 0}\left(z \mid \begin{array}{cc}
- & - \\
b_{1}, b_{2}, \ldots, b_{m} & -
\end{array}\right) \\
& =\frac{1}{2 \pi i} \int_{L} \Gamma\left(b_{1}-\zeta\right) \Gamma\left(b_{2}-\zeta\right) \ldots \Gamma\left(b_{m}-\zeta\right) z^{\zeta} d \zeta
\end{aligned}
$$

We recall selected basic formulae involving Meijer $G$-functions (see [1], [2], [7]). By the definition of $G$-function we have

$$
\begin{aligned}
& z^{\sigma} G_{p, q}^{m, n}\left(z \mid \begin{array}{l}
a_{1}, \ldots, a_{p} \\
b_{1}, \ldots, b_{q}
\end{array}\right)=G_{p, q}^{m, n}\left(z \mid \begin{array}{l}
a_{1}+\sigma, \ldots, a_{p}+\sigma \\
b_{1}+\sigma, \ldots, b_{q}+\sigma
\end{array}\right)
\end{aligned}
$$

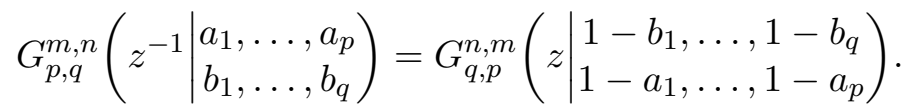

The classical integral formula for Meijer $G$-functions is of fundamental importance in calculations:

$$
\begin{aligned}
& \int_{0}^{\infty} G_{p, q}^{m, n}\left(\eta x \mid \begin{array}{l}
a_{1}, \ldots, a_{p} \\
b_{1}, \ldots, b_{q}
\end{array}\right) G_{\sigma, \tau}^{\mu, \nu}\left(\omega x \mid \begin{array}{l}
c_{1}, \ldots, c_{\sigma} \\
d_{1}, \ldots, d_{\tau}
\end{array}\right) d x \\
= & \frac{1}{\eta} G_{q+\sigma, p+\tau}^{n+\mu, m+\nu}\left(\frac{\omega}{\eta} \mid \begin{array}{l}
-b_{1}, \ldots,-b_{m}, c_{1}, \ldots, c_{\sigma},-b_{m+1}, \ldots,-b_{q} \\
-a_{1}, \ldots,-a_{n}, d_{1}, \ldots, d_{\tau},-a_{n+1}, \ldots,-a_{p}
\end{array}\right)
\end{aligned}
$$

By (3) and (4) we get a slightly more general integral formula

$$
\begin{aligned}
& \int_{0}^{\infty} x^{\alpha-1} G_{p, q}^{m, n}\left(\eta x \mid \begin{array}{l}
a_{1}, \ldots, a_{p} \\
b_{1}, \ldots, b_{q}
\end{array}\right) G_{\sigma, \tau}^{\mu, \nu}\left(\omega x \mid \begin{array}{l}
c_{1}, \ldots, c_{\sigma} \\
d_{1}, \ldots, d_{\tau}
\end{array}\right) d x \\
& =\eta^{1-\alpha} \int_{0}^{\infty} G_{p, q}^{m, n}\left(\eta x \mid \begin{array}{l}
a_{1}+\alpha-1, \ldots, a_{p}+\alpha-1 \\
b_{1}+\alpha-1, \ldots, b_{q}+\alpha-1
\end{array}\right) G_{\sigma, \tau}^{\mu, \nu}\left(\omega x \mid \begin{array}{l}
c_{1}, \ldots, c_{\sigma} \\
d_{1}, \ldots, d_{\tau}
\end{array}\right) d x \\
& =\eta^{-\alpha} G_{q+\sigma, p+\tau}^{n+\mu, m+\nu}\left(\frac{\omega}{\eta} \mid \begin{array}{l}
-b_{1}-\alpha+1, \ldots,-b_{m}-\alpha+1, c_{1}, \ldots, c_{\sigma},-b_{m+1}-\alpha+1, \ldots,-b_{q}-\alpha+1 \\
-a_{1}-\alpha+1, \ldots,-a_{n}-\alpha+1, d_{1}, \ldots, d_{\tau},-a_{n+1}-\alpha+1, \ldots,-a_{p}-\alpha+1
\end{array}\right)
\end{aligned}
$$

By (3) and (5) we have

$$
\begin{aligned}
& \int_{0}^{\infty} x^{\alpha-1} G_{p, q}^{m, n}\left(\eta x \mid \begin{array}{l}
a_{1}, \ldots, a_{p} \\
b_{1}, \ldots, b_{q}
\end{array}\right) G_{\sigma, \tau}^{\mu, \nu}\left(\frac{1}{\omega x} \mid \begin{array}{l}
c_{1}, \ldots, c_{\sigma} \\
d_{1}, \ldots, d_{\tau}
\end{array}\right) d x \\
& =\int_{0}^{\infty} x^{\alpha-1} G_{p, q}^{m, n}\left(\eta x \mid \begin{array}{l}
a, \ldots, a_{p} \\
b_{1}, \ldots, b_{q}
\end{array}\right) G_{\tau, \sigma}^{\nu, \mu}\left(\omega x \mid \begin{array}{l}
1-d_{1}, \ldots, 1-d_{\tau} \\
1-c_{1}, \ldots, 1-c_{\sigma}
\end{array}\right) d x \\
& =\eta^{-\alpha} G_{q+\tau, p+\sigma}^{n+\nu, m+\mu}\left(\frac{\omega}{\eta} \mid \begin{array}{l}
-b_{1}-\alpha+1, \ldots,-b_{m}-\alpha+1,1-d_{1}, \ldots, 1-d_{\tau},-b_{m+1}-\alpha+1, \ldots,-b_{q}-\alpha+1 \\
-a_{1}-\alpha+1, \ldots,-a_{n}-\alpha+1,1-c_{1}, \ldots, 1-c_{\sigma},-a_{n+1}-\alpha+1, \ldots,-a_{p}-\alpha+1
\end{array}\right) \\
& =\eta^{-\alpha} G_{p+\sigma, q+\tau}^{m+\mu, n+\nu}\left(\frac{\eta}{\omega} \mid \begin{array}{l}
a_{1}+\alpha, \ldots, a_{n}+\alpha, c_{1}, \ldots, c_{\sigma}, a_{n+1}+\alpha, \ldots, a_{p}+\alpha \\
b_{1}+\alpha, \ldots, b_{m}+\alpha, d_{1}, \ldots, d_{\tau}, b_{m+1}+\alpha, \ldots, b_{q}+\alpha
\end{array}\right)
\end{aligned}
$$

Putting $\alpha=0$ in (6) we get

$$
\begin{aligned}
& \int_{0}^{\infty} G_{p, q}^{m, n}\left(\eta x \mid \begin{array}{l}
a_{1}, \ldots, a_{p} \\
b_{1}, \ldots, b_{q}
\end{array}\right) G_{\sigma, \tau}^{\mu, \nu}\left(\frac{1}{\omega x} \mid \begin{array}{c}
c_{1}, \ldots, c_{\sigma} \\
d_{1}, \ldots, d_{\tau}
\end{array}\right) \frac{d x}{x} \\
= & G_{p+\sigma, q+\tau}^{m+\mu, n+\nu}\left(\frac{\eta}{\omega} \mid \begin{array}{l}
a_{1}, \ldots, a_{n}, c_{1}, \ldots, c_{\sigma}, a_{n+1}, \ldots, a_{p} \\
b_{1}, \ldots, b_{m}, d_{1}, \ldots, d_{\tau}, b_{m+1}, \ldots, b_{q}
\end{array}\right)
\end{aligned}
$$


For $\omega=t^{-1}$ and $\eta=1$ in (7) we obtain the multiplicative convolution formula for Meijer G-functions:

$$
\begin{aligned}
\left\{G_{p, q}^{m, n} * G_{\sigma, \tau}^{\mu, \nu}\right\}(t) & :=\int_{0}^{\infty} G_{p, q}^{m, n}(x) G_{\sigma, \tau}^{\mu, \nu}\left(t x^{-1}\right) \frac{d x}{x} \\
& =G_{p+\sigma, q+\tau}^{m+\mu, n+\nu}(t)
\end{aligned}
$$

or more specifically

$$
\begin{aligned}
& \int_{0}^{\infty} G_{p, q}^{m, n}\left(x \mid \begin{array}{l}
a_{1}, \ldots, a_{p} \\
b_{1}, \ldots, b_{q}
\end{array}\right) G_{\sigma, \tau}^{\mu, \nu}\left(\frac{t}{x} \mid \begin{array}{c}
c_{1}, \ldots, c_{\sigma} \\
d_{1}, \ldots, d_{\tau}
\end{array}\right) \frac{d x}{x} \\
= & G_{p+\sigma, q+\tau}^{m+\mu, n+\nu}\left(t \mid \begin{array}{l}
a_{1}, \ldots, a_{n}, c_{1}, \ldots, c_{\sigma}, a_{n+1}, \ldots, a_{p} \\
b_{1}, \ldots, b_{m}, d_{1}, \ldots, d_{\tau}, b_{m+1}, \ldots, b_{q}
\end{array}\right)
\end{aligned}
$$

By (6) we get also

$$
\begin{aligned}
& \int_{0}^{\infty} x^{\alpha-1} G_{p, q}^{m, n}\left(\frac{\eta x^{2}}{2} \mid \begin{array}{l}
a_{1}, \ldots, a_{p} \\
b_{1}, \ldots, b_{q}
\end{array}\right) G_{\sigma, \tau}^{\mu, \nu}\left(\frac{2}{\omega x^{2}} \mid \begin{array}{c}
c_{1}, \ldots, c_{\sigma} \\
d_{1}, \ldots, d_{\tau}
\end{array}\right) d x \\
= & 2^{\frac{\alpha}{2}-1} \int_{0}^{\infty} s^{\frac{\alpha}{2}-1} G_{p, q}^{m, n}\left(\eta \mid \begin{array}{l}
a_{1}, \ldots, a_{p} \\
b_{1}, \ldots, b_{q}
\end{array}\right) G_{\sigma, \tau}^{\mu, \nu}\left(\frac{1}{\omega s} \mid \begin{array}{c}
c_{1}, \ldots, c_{\sigma} \\
d_{1}, \ldots, d_{\tau}
\end{array}\right) d s \\
= & 2^{\frac{\alpha}{2}-1} \eta^{-\frac{\alpha}{2}} G_{p+\sigma, q+\tau}^{m+\mu, n+\nu}\left(\frac{\eta}{\omega} \mid \begin{array}{l}
a_{1}+\alpha, \ldots, a_{n}+\alpha, c_{1}, \ldots, c_{\sigma}, a_{n+1}+\alpha, \ldots, a_{p}+\alpha \\
b_{1}+\alpha, \ldots, b_{m}+\alpha, d_{1}, \ldots, d_{\tau}, b_{m+1}+\alpha, \ldots, b_{q}+\alpha
\end{array}\right)
\end{aligned}
$$

Putting $\alpha=0, \eta=1$ and $\omega=t^{-1}$ in (9) we get

$$
\begin{aligned}
& \int_{0}^{\infty} x^{\alpha-1} G_{p, q}^{m, n}\left(\frac{x^{2}}{2} \mid \begin{array}{c}
a_{1}, \ldots, a_{p} \\
b_{1}, \ldots, b_{q}
\end{array}\right) G_{\sigma, \tau}^{\mu, \nu}\left(\frac{2 t}{x^{2}} \mid \begin{array}{l}
c_{1}, \ldots, c_{\sigma} \\
d_{1}, \ldots, d_{\tau}
\end{array}\right) d x \\
= & \frac{1}{2} G_{p+\sigma, q+\tau}^{m+\mu, n+\nu}\left(t \mid \begin{array}{l}
a_{1}, \ldots, a_{n}, c_{1}, \ldots, c_{\sigma}, a_{n+1}, \ldots, a_{p} \\
b_{1}, \ldots, b_{m}, d_{1}, \ldots, d_{\tau}, b_{m+1}, \ldots, b_{q}
\end{array}\right)
\end{aligned}
$$

We recall a formula for the $G_{0,2}^{2,0}$-function

$$
G_{0,2}^{2,0}(x \mid a, b)=G_{0,2}^{2,0}\left(x \mid \begin{array}{cc}
- & - \\
a, b & -
\end{array}\right)=2 x^{\frac{a+b}{2}} K_{a-b}(2 \sqrt{x})
$$

(see 5.6(4) in [1]), where $K_{\nu}$ is the modified Bessel function of the second kind of order $\nu$. Many other formulae to express the Meijer $G$-function in other special functions can be found in [1]. Most likely, we should not expect, unfortunately, a reasonable reduction of the Meijer function $G_{0, m}^{m, 0}$ to simpler special functions when $m>2$. In [3] some applications of the Fox $H$ function

$$
H_{p, q}^{m, n}\left[z \mid \begin{array}{c}
\left(a_{1}, \alpha_{1}\right), \ldots,\left(a_{p}, \alpha_{p}\right) \\
\left(b_{1}, \beta_{1}\right), \ldots,\left(b_{q}, \beta_{q}\right)
\end{array}\right]:=\frac{1}{2 \pi i} \int_{L} \frac{\prod_{k=1}^{m} \Gamma\left(b_{k}-\beta_{k} \zeta\right)}{\prod_{k=m+1}^{q} \Gamma\left(1-b_{k}+\beta_{k} \zeta\right)} \frac{\prod_{k=1}^{n} \Gamma\left(1-a_{k}+\alpha_{k} \zeta\right)}{\prod_{k=n+1}^{p} \Gamma\left(a_{k}-\alpha_{k} \zeta\right)} z^{\zeta} d \zeta
$$


were investigated to study the distribution of products, quotients and powers of independent random variables. However the Fox $H$ function generalizes the Meijer $G$ function and we should not expect all the more any simplification of results formulated in terms of the Fox $H$ function to more elementary functions.

\section{Main result}

Theorem 3.1 Let $\xi_{1}, \xi_{2}, \ldots, \xi_{m}$ be i.i.d. random variables with standard normal p.d.f. with $m_{i}=0$ and $\sigma_{i}=1$, see (1). Then the product $\xi_{1} \xi_{2} \ldots \xi_{m}$ has p.d.f. given by

$$
\begin{aligned}
f_{\xi_{1} \xi_{2} \ldots \xi_{m}}(t) & =(2 \pi)^{-m / 2} G_{0, m}^{m, 0}(2^{-m} t^{2} \mid \underbrace{0,0, \ldots, 0}_{m \text { parameters }}) \\
& =\frac{1}{(\sqrt{2 \pi})^{m}} G_{0, m}^{m, 0}\left(\frac{t^{2}}{2^{m}} \mid \begin{array}{cc}
- & - \\
0,0, \ldots, 0 & -
\end{array}\right)
\end{aligned}
$$

In the case when $m=2$ we have

$$
f_{\xi_{1} \xi_{2}}(t)=\frac{1}{\pi} K_{0}(|t|)
$$

where $K_{0}$ is the modified Bessel function of the second kind of order 0 .

Proof. By the formula for the p.d.f. of the product $\xi \eta$ of two independent random variables $\xi$ and $\eta$ with p.d.f. $f_{\xi}$ and $f_{\eta}$, respectively, we get (see eg. $[6])$ :

$$
\begin{aligned}
f_{\xi \eta}(t) & =\int_{-\infty}^{\infty} f_{\xi}\left(t x^{-1}\right) f_{\eta}(x) \frac{d x}{|x|} \\
& =\int_{-\infty}^{\infty} f_{\xi}(x) f_{\eta}\left(t x^{-1}\right) \frac{d x}{|x|}
\end{aligned}
$$

Hence for two i.i.d. random variables $\xi_{1}, \xi_{2}$ with standard normal p.d.f. we have

$$
f_{\xi_{1} \xi_{2}}(t)=\int_{-\infty}^{\infty} f_{\xi_{1}}\left(t x^{-1}\right) f_{\xi_{2}}(x) \frac{d x}{|x|}=2 \int_{0}^{\infty} f_{\xi_{1}}\left(t x^{-1}\right) f_{\xi_{2}}(x) \frac{d x}{x}
$$

because the function $f_{\xi_{2}}$ is even. The last integral is the multiplicative convolution of functions $f_{\xi_{1}}$ and $f_{\xi_{2}}$ :

$$
\int_{0}^{\infty} f_{\xi_{1}}\left(t x^{-1}\right) f_{\xi_{2}}(x) \frac{d x}{x}=:\left\{f_{\xi_{1}} * f_{\xi_{2}}\right\}(t) .
$$


By the convolution formula for Meijer $G$-functions, see (8):

$$
G_{p, q}^{m, n} * G_{p^{\prime}, q^{\prime}}^{m^{\prime}, n^{\prime}}=G_{p+p^{\prime}, q+q^{\prime}}^{m+m^{\prime}, n+n^{\prime}}
$$

for $f_{\xi_{1}}(u)=f_{\xi_{2}}(u)=\frac{1}{\sqrt{2 \pi}} \exp \left(-\frac{1}{2} u^{2}\right)=\frac{1}{\sqrt{2 \pi}} G_{0,1}^{1,0}\left(\left.\frac{u^{2}}{2}\right|_{0} ^{-} \quad-\quad\right)=\frac{1}{\sqrt{2 \pi}} G_{0,1}^{1,0}\left(\frac{u^{2}}{2} \mid 0\right)$

and using formulae (10) and (11) we get:

$$
\begin{aligned}
& f_{\xi_{1} \xi_{2}}(t)=2 \int_{0}^{\infty} f_{\xi_{1}}\left(t x^{-1}\right) f_{\xi_{2}}(x) \frac{d x}{x} \\
& =\frac{2}{(\sqrt{2 \pi})^{2}} \int_{0}^{\infty} G_{0,1}^{1,0}\left(\frac{1}{2} \frac{t^{2}}{x^{2}} \mid \begin{array}{ll}
- & - \\
0 & -
\end{array}\right) G_{0,1}^{1,0}\left(\frac{x^{2}}{2} \mid \begin{array}{ll}
- & - \\
0 & -
\end{array}\right) \frac{d x}{x}
\end{aligned}
$$

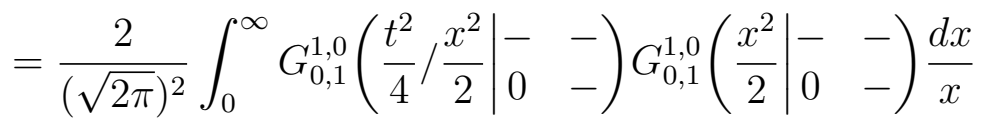

$$
\begin{aligned}
& =\frac{2}{(\sqrt{2 \pi})^{2}} \cdot \frac{1}{2} G_{0,2}^{2,0}\left(\frac{t^{2}}{4} \mid \begin{array}{cc}
- & - \\
0,0 & -
\end{array}\right)=\frac{1}{2 \pi} G_{0,2}^{2,0}\left(2^{-2} t^{2} \mid 0,0\right)=\frac{1}{\pi} K_{0}(|t|)
\end{aligned}
$$

Assume for $m \geq 2$ the product $\xi_{1} \xi_{2} \ldots \xi_{m-1}$ has p.d.f. of the form

$$
f_{\xi_{1} \xi_{2} \ldots \xi_{m-1}}(t)=\frac{1}{(\sqrt{2 \pi})^{m-1}} G_{0, m-1}^{m-1,0}(\frac{t^{2}}{2^{m-1}} \mid \underbrace{0, \ldots, 0}_{m-1 \text { parameters }}-)
$$

Proceeding as above for two factors $f_{\xi_{1}}$ and $f_{\xi_{2}}$ we have by (10):

$$
\begin{aligned}
& f_{\xi_{1} \xi_{2} \ldots \xi_{m-1} \xi_{m}}(t)=2 \int_{0}^{\infty} f_{\xi_{2} \xi_{2} \ldots \xi_{m}}\left(t x^{-1}\right) f_{\xi_{1}}(x) \frac{d x}{x} \\
& =\frac{2}{(\sqrt{2 \pi})^{m}} \int_{0}^{\infty} G_{0, m-1}^{m-1,0}\left(\frac{1}{2^{m-1}} \frac{t^{2}}{x^{2}} \mid \begin{array}{ccc}
0, \ldots, 0 & -
\end{array}\right) G_{0,1}^{1,0}\left(\frac{x^{2}}{2} \mid \begin{array}{cc}
- & - \\
0 & -
\end{array}\right) \frac{d x}{x} \\
& =\frac{2}{(\sqrt{2 \pi})^{m}} \int_{0}^{\infty} G_{0, m-1}^{m-1,0}\left(\frac{t^{2}}{2^{m}} /\left.\frac{x^{2}}{2}\right|_{0, \ldots, 0}-\begin{array}{cc}
- & -
\end{array}\right) G_{0,1}^{1,0}\left(\frac{x^{2}}{2} \mid \begin{array}{ll}
- & - \\
0 & -
\end{array}\right) \frac{d x}{x} \\
& =\frac{2}{(\sqrt{2 \pi})^{m}} \cdot \frac{1}{2} G_{0, m}^{m, 0}(\frac{t^{2}}{2^{m}} \mid \underbrace{0, \ldots, 0,0}_{m \text { parameters }}-) \\
& =\frac{1}{(\sqrt{2 \pi})^{m}} G_{0, m}^{m, 0}\left(2^{-m} t^{2} \mid 0,0, \ldots, 0\right) \text {. }
\end{aligned}
$$

Corrolary 3.2 Let $\xi_{1}, \xi_{2}, \ldots, \xi_{m}$ be independent normally distributed random variables with mean values $m_{i}=0$ and standard deviations $\sigma_{i}>0$, i.e. 
with p.d.f.

$$
f_{\xi_{i}}(t)=\frac{1}{\sigma_{i} \sqrt{2 \pi}} \exp \left(-\frac{t^{2}}{\sigma_{i}^{2}}\right), i=1,2, \ldots, m .
$$

The p.d.f. of the product $\xi_{1} \xi_{2} \ldots \xi_{m}$ equals

$$
f_{\xi_{1} \xi_{2} \ldots \xi_{m}}(t)=\frac{1}{\sigma(\sqrt{2 \pi})^{m}} G_{0, m}^{m, 0}\left(2^{-m}(t / \sigma)^{2} \mid 0, \ldots, 0\right)
$$

where $\sigma:=\sigma_{1} \sigma_{2} \ldots \sigma_{m}$.

Remark 3.3 It was noticed in [4], [5] that the density (14) of the product $\xi_{1} \xi_{2}$ was found in 1932 in [11]. The formula (14) is also an exercise to the reader in [8]. The density (15) of the product $\xi_{1} \xi_{2} \ldots \xi_{m}$ was found in [9] in 1966. See also [10].

Question 3.4 Is it possible to simplify the Meijer function $G_{0, m}^{m, 0}(x \mid 0, \ldots, 0)$ for $m>2$ as in the case of $m=2$ when $G_{0,2}^{2,0}\left(\frac{t^{2}}{4} \mid 0,0\right)=2 K_{0}(|t|)$ ?

Question 3.5 Is it possible to give an explicit formula for the p.d.f. of the product $\xi_{1} \xi_{2} \ldots \xi_{m}, m \geq 2$, of normally distributed independent random variables with different mean values?

We may find a partial answer to the question in [8] in a special case of the product $\xi_{1} \xi_{2}$ where $\xi_{1} \sim N(0, \sigma)$ and $\xi_{2} \sim N(m, \sigma)$.

\section{Acknowledgements}

The author would like to thank the reviewers for drawing attention to the results published in [3], [4], [5], [8], [9], [10], [11] and for indicating an error in the formula (5) in the earlier version of the paper.

\section{References}

1. Bateman H, Erdélyi A. Higher transcendental functions. Vol. 1. New York: McGraw-Hill; 1953.

2. Beals R, Szmigielski J. Meijer G-functions: a gentle introduction. Notices of the American Mathematical Society. 2013;60(7):866-872. doi: http://dx.doi.org/10.1090/ notimanid 1016 .

3. Carter BD, Springer MD. The distribution of products, quotients and powers of independent $\mathrm{H}$-function variates. SIAM Journal on Applied Mathematics. 1977;33(4):542558. doi: https://doi.org/10.1137/0133036.
4. Craig CC. On the frequency function of XY. The Annals of Mathematical Statistics. 1936;7(1):1-15.

5. Craig CC. On frequency distributions of the quotient and of the product of two statistical variables. The American Mathematical Monthly. 1942;49(1):24-32. doi: https://doi. org/10.1080/00029890.1942.11991175.

6. Fisz M. Probability and Mathematical Statistics. Delhi: S. Chand; 1961.

7. Gradshteyn IS, Ryzhik IM. Table of Integrals, Series, and Products. 7th ed. Amsterdam: Academic Press; Elsevier; 2007.

8. Springer MD. The Algebra of Random Variables. Wiley Series in Probability and Mathematical Statistics. New York-Chichester-Brisbane: John Wiley \& Sons; 1979.

9. Springer MD, Thompson WE. The distribution of products of independent random variables. SIAM Journal on Applied Mathematics. 1966;14(3):511-526. doi: https:// doi.org/10.1137/0114046.

10. Springer MD, Thompson WE. The distribution of products of beta, gamma and Gaussian random variables. SIAM 
Journal on Applied Mathematics. 1970;18(4):721-737. doi: https://doi.org/10.1137/0118065.

11. Wishart J, Barlett MS. The distribution of second order moment statistics in a normal system. Mathematical
Proceedings of the Cambridge Philosophical Society. 1932;28(4):455-459. doi: https://doi.org/10.1017/ S0305004100010690.
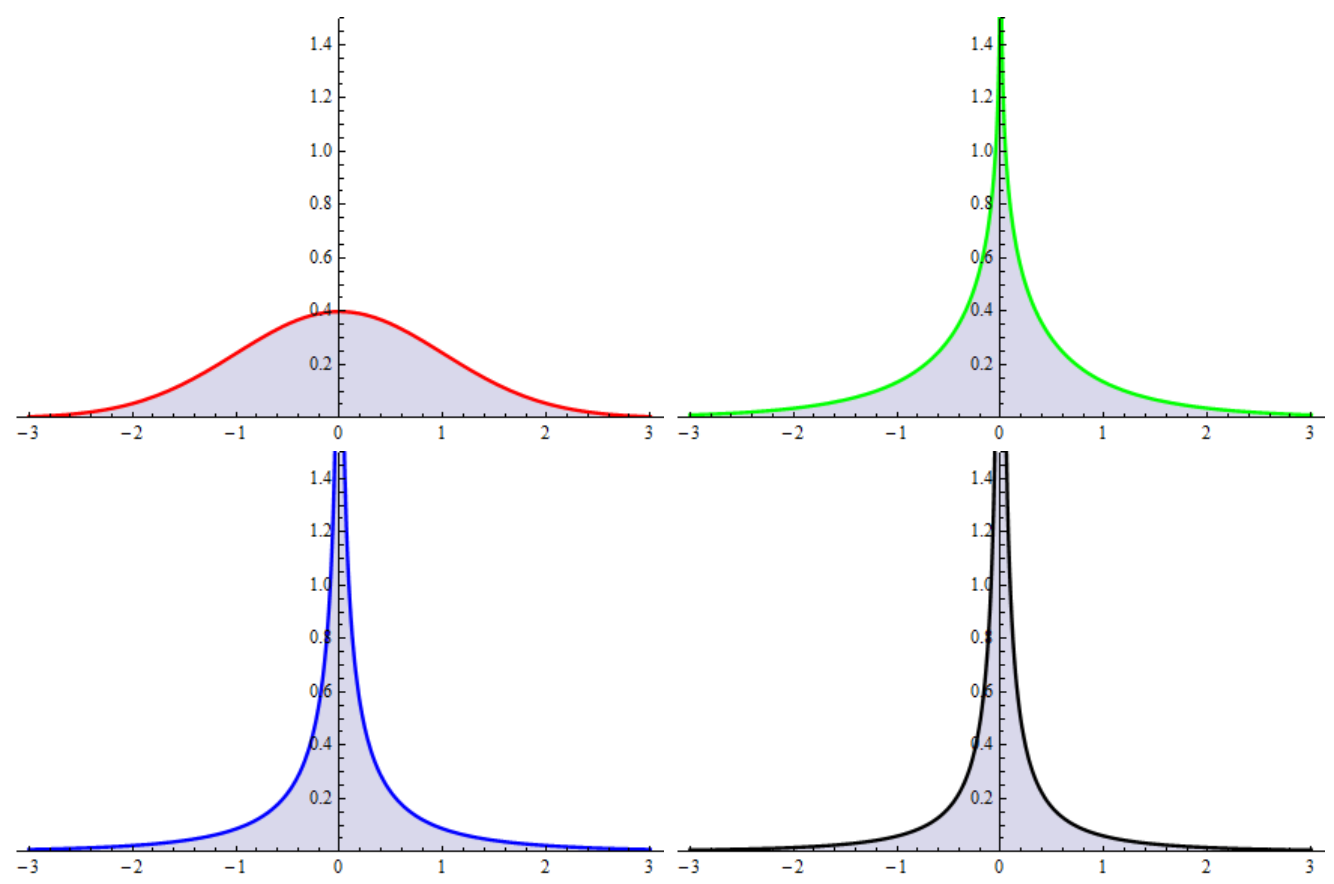

Figure 1: Plots of the p.d.f. of the products $\xi_{1} \ldots \xi_{m}$ for $m=1$ (red line), $m=2$ (green line), $m=3$ (blue line) and $m=4$ (black line).

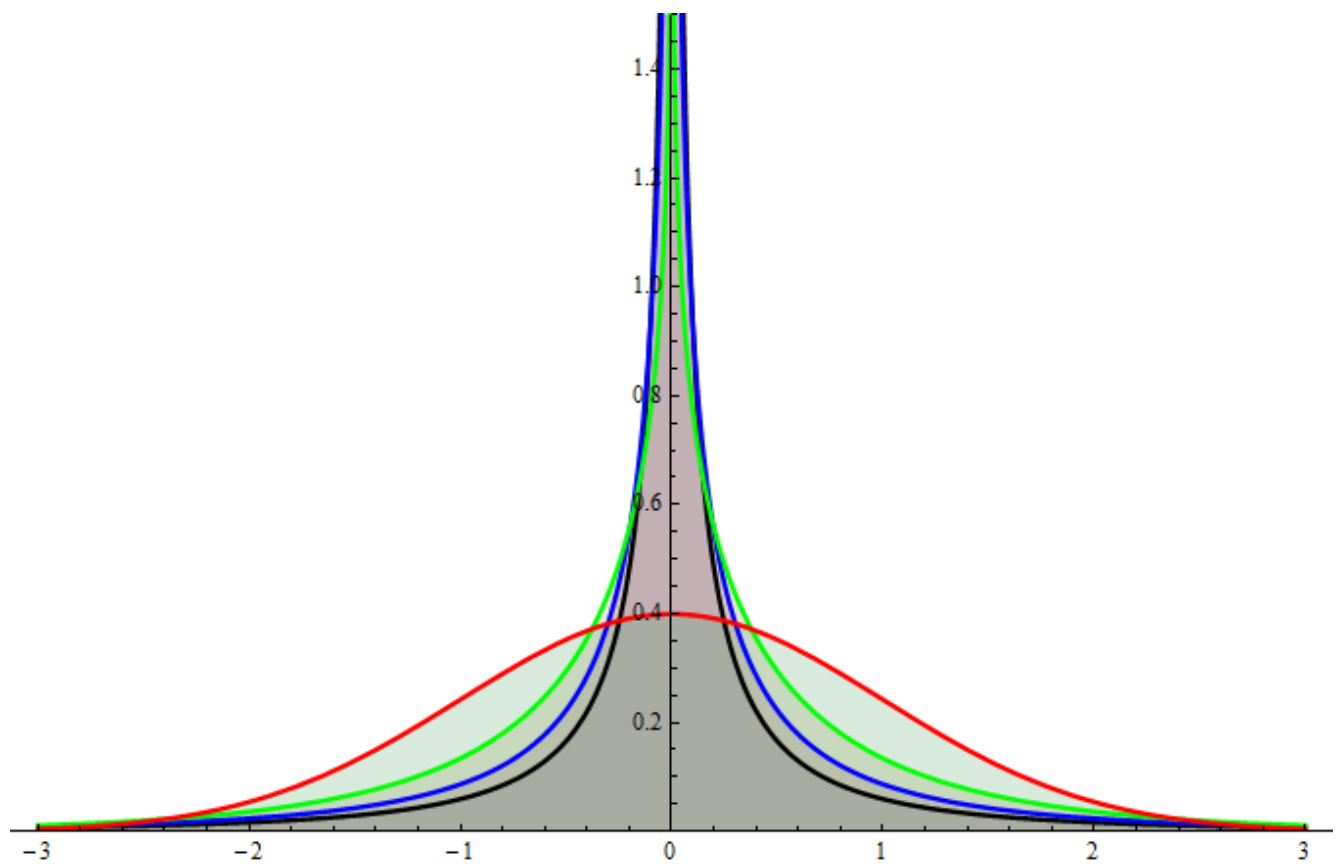

Figure 2: Plots of the p.d.f. of the products $\xi_{1} \ldots \xi_{m}$ for $m=1$ (red line), $m=2$ (green line). $m=3$ (blue line) and $m=4$ (black line). 